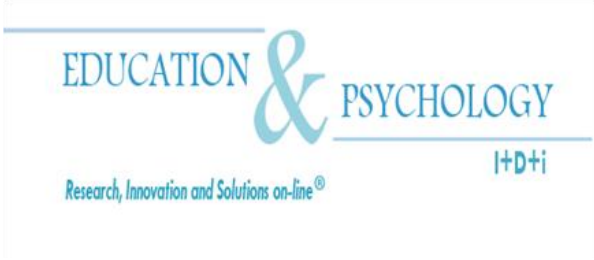

\title{
Utilidad del cuestionario CUMANIN para detectar diferencias, en dos grupos de preescolares, dentro de un programa de Atención Temprana
}

\author{
Francisca Barba Colmenero y \\ María Auxiliadora Robles Bello
}

Departamento de Psicología. Universidad de Jaén, Jaén

\section{España}

Correspondencia: Francisca Barba Colmenero. Asociación Provincial de Autismo y/o TGD de Jaén "Juan Martos Pérez" Avenida de Andalucía, 92 bajo 23006 Jaén. España. E-mail: paqui.barba@ autismojaen.es marobles@ujaen.es

(C) Education \& Psychology I+D+i and Editorial EOS (Spain) 


\section{Resumen}

Introducción. El presente estudio busca las diferencias existentes a nivel neuropsicológico, observando las discrepancias en el lenguaje en niños con Síndrome de Down y niños con Dificultades de Aprendizaje.

Método. Para ello se ha utilizado el Cuestionario de Madurez Neuropsicológica (CUMANIN) en una población de 51 niños, de edades comprendidas entre los 3 y 6 años, pertenecientes a un programa de Atención Temprana, de La Asociación Síndrome de Down Jaén (España) divididos en dos grupos, Síndrome de Down y niños con Dificultades del Aprendizaje y 29 niños sin diagnóstico pertenecientes a centros de educación de la provincia de Jaén.

Resultados. Los resultados encontrados indican que existen diferencias significativas entre niños que reciben terapia en la asociación y niños sin diagnostico. Además, no se han encontrando diferencias entre niños con Síndrome de Down y niños con Dificultades de Aprendizaje que reciben terapia en el mismo centro, en la mayoría de las variables, entre ellas el lenguaje.

Discusión y conclusión. Se concluye que ambos grupos de niños presentan las mismas limitaciones en el lenguaje o, bien, que la escala no discrimina entre ellos. Por tanto, se puede avanzar en el estudio de niños con problemas del lenguaje y planificar tratamientos más eficaces, que puedan beneficiarlos.

Palabras Clave: Atención Temprana. Dificultades de Aprendizaje. Desarrollo. Lenguaje. Síndrome de Down. 


\title{
Utility of the CUMANIN questionnaire for detecting differences in two groups of preschool aged children in an Early Care program
}

\begin{abstract}
Introduction. This study looks for differences in neuropsychological level by obtaining the differences in language between children with Down syndrome and children with Learning Disabilities.
\end{abstract}

Method. For this analysis we have used the Neuropsychological Maturity Questionnaire (CUMANIN) in a population of 51 children, aged between 3 and 6 years old. They are attending an early childhood program of the Down Syndrome Association of Jaén (Spain) divided into two groups, Down Syndrome and children with Learning Disabilities and 29 children without a diagnosis pertaining to education centres of the province of Jaén.

Results. The obtained results have shown significant differences between children from this association and the children without diagnosis. In addition, no differences have been found between children with Down syndrome and children with learning disabilities who have received therapy at this centre in the majority of the variables, including language.

Discussion and Conclusion. We conclude that both groups of children have the same constraints in language or that the used scale does not discriminate between them. Therefore, it can advance the study of children with language problems and in planning more effective treatments which can benefit them.

Keywords: Early Care; Learning Disabilities; development; language; Down syndrome. 


\section{Introducción}

Cuando se hace referencia a lenguaje en niños con Síndrome de Down, es importante conocer las características lingüísticas específicas, si se compara con otros niños que tienen otras dificultades de aprendizaje. El conocimiento de estas características permitirá elegir un tratamiento u otro, y por tanto su eficacia, además de reforzar la importancia de la neuropsicología infantil, especialmente entre los tres y seis años, periodo en el que se observa un amplio desarrollo de las funciones cognitivas.

A la hora de clasificar a los diferentes trastornos o disfunciones que se pueden encontrar en Atención Temprana (en adelante AT), los ODAT (Organización Diagnostica para la Atención Temprana, 2008) clasifica a los niños en seis ejes. Esta clasificación no tiene una categoría que discrimine a los niños con dificultades del aprendizaje (en adelante DA), pero en el eje IV, en los trastornos del desarrollo, existe una categoría para los trastornos en el desarrollo de la comunicación y del lenguaje en la que incluiremos a los niños con DA, ya que así aparece en su historia clínica que aporta el neuropediatra. El término Trastornos en el Desarrollo de la Comunicación y del Lenguaje engloba a un conjunto de niños con grandes diferencias entre sí dada la naturaleza multidimensional del lenguaje (Hasson y Joffe, 2007). De hecho, en este grupo a veces se incluyen niños con diferencias lingüísticas o culturales o pertenecientes a grupos desfavorecidos socialmente junto con niños con problemas de diferentes aspectos de lenguaje o con problemas de lectura. Respecto a las dificultades que conlleva la evaluación psicológica de este grupo de niños cabe destacar la irrelevancia del C.I. en su diagnóstico y predicción de su desarrollo -pues los problemas de lenguaje no suelen correlacionar con el C.I.- (Swanson y Howard, 2005) y la escasa fiabilidad que poseen las medidas de screening lingüístico al uso para establecer el problema y para indicar el tratamiento (Hasson y Joffe, 2007).

Tanto la definición como la clasificación de los DA está llena de controversia y ni los investigadores ni los profesionales se ponen de acuerdo en definirla. Aún así, coinciden en que estos niños, muestran problemas tanto en el logro académico como en su potencial intelectual, en uno o más de los siguientes: comprensión oral, expresión oral, habilidades básicas de lectura y su comprensión, expresión escrita, calculo y razonamiento matemático (Miranda, Vidal-Abarca y Soriano, 2000). La definición del manual diagnóstico y estadístico de los trastornos mentales DSM IV-TR. Indica: "Las dificultades de aprendizaje se diagnostican cuando el rendimiento del individuo en lectura, calculo o expresión escrita es sustancialmente inferior 
al esperado por la edad, escolarización y nivel de inteligencia". Se puede afirmar que, además las DA vienen definidas por la existencia de algún tipo de disfunción del sistema nervioso, que ya se puede observar mucho antes a la aparición de los problemas de aprendizaje de la etapa escolar. Las DA están asociadas a otros problemas no relacionados con áreas académicas: área social, área personal, así como el área conductual. (Miranda, Vidal-Abarca y Soriano, 2000).

Bisquerra (2000) entiende por DA las que "afectan a la comprensión o al uso del lenguaje, y se manifiestan en el habla, lectura escritura y cálculo" y hay una discrepancia manifiesta entre el potencial del alumno y su rendimiento. Pueden existir junto con las DA un conjunto de trastornos pero no constituyen en sí mismos DA y no son el resultado de estas condiciones o influencias, por lo que pueden excluirse según ese autor los bajos rendimientos explicables por déficits sensoriales, discapacidad intelectual, alteraciones sociales y/o emocionales graves o condiciones extrínsecas, como diferencias culturales o ausencia de oportunidades educativas. Se le critica su dificultad para diferenciar estudiantes con dificultades de aprendizaje y estudiantes con bajo rendimiento. Según todo ello las dificultades son inesperadas: existe discrepancia entre potencial y rendimiento (entre lo esperado y lo observado), su CI se puede encontrar por encima o debajo de la media, además existen trastornos que se manifiestan específicamente en problemas del lenguaje, razonamiento y aprendizajes instrumentales básicos. Este criterio es útil para diferenciar DA de bajo rendimiento: un alumno con DA tendrá dificultades en un área específica, siendo su rendimiento en otras áreas normal. DA en dominios, cognitivos y académicos: lenguaje, razonamiento o aprendizajes básicos.

Según Padget (1998) se clasifica a las DA en tres: dificultades especificas en el lenguaje, dificultades específicas en la lectura y dificultades específicas en las matemáticas. Este estudio, se va a centrar más detenidamente en las dificultades que presentan los DA en el lenguaje. En los niños con DA, las dificultades especificas del lenguaje, están asociadas a los problemas en el vocabulario y sintaxis. Todo ello afecta al desarrollo de la comunicación oral, en la comprensión del lenguaje escrito y al contenido de la expresión escrita (Miranda, VidalAbarca y Soriano, 2000).

Otro de los grupos del presente estudio son los niños con Síndrome de Down (en adelante SD) y los ODAT los clasifica dentro del eje 1: Factores biológicos de riesgo, de origen prenatal y de tipo cromosómico. Estos niños presentan un cromosoma 21 de más y la consecuente dotación genética anormal, provocando un desequilibrio que condiciona las alteracio- 
nes del desarrollo, estructura y función de diversos sistemas y órganos. El resultado de estos desequilibrios son los diversos tipos y grados de disfunción cognitiva y neurológica que presentan (Rondal, Perera, Nadel y Comblain, 1997).

A nivel cognitivo, en general se puede afirmar que el desarrollo es mucho más lento y el aprendizaje está más deteriorado, afectando a la memoria, atención y al lenguaje. Hay indicios que sugieren que este deterioro no se extiende por todos los sistemas de aprendizaje y de la memoria, más bien afecta a algunas zonas implicadas, (hipocampo y cortex entorrinal), (Rondal, Perera, Nadel y Comblain, 1997). Esto da lugar a que este retraso se puede observar ya desde los 0 y los 2 años de edad, y cada vez más acusado durante los 2 y 4 años (Chapman y Hesketh, 2000).

En los procesos atencionales, los niños más pequeños muestran problemas a la hora de fijar la mirada, debido a que su dificultad de mantener la cabeza por la laxitud ligamentosa y por el bajo tono muscular (Flórez y Ruiz, 2004). En las primeras etapas la atención auditiva parece mejorar, aunque con problemas de percepción y discriminación. Esto dificulta que el niño no escuche o no atienda, llegando a preferir acciones manipulativas (Flórez y Ruiz 2004). Por ello son muchos los autores y estudios que confirman la idea de que estos niños procesan mejor la información visual que la auditiva (Calero, Robles y García, 2010).

En niños más mayores se acentúan las dificultades para mantener la atención, muestran menor motivación para enfrentarse con deseo al aprendizaje, evitan enfrentarse a situaciones de aprendizaje complejas auditiva-verbal y presentan lentitud en los tiempos de reacción. Tienen dificultades en la comprensión de conceptos como los números, y son incapaces de formar conceptos, además de dificultades para agrupar objetos en categorías superiores con significado (Arregi, 2007). En estos niños se aprecia un deterioro en tareas que requieran nivel de abstracción, problemas de cálculo matemático, problemas en la adquisición del simbolismo y su manipulación, Además presentan dificultades a la hora de general conocimientos y su aplicación a los contextos y ámbitos fuera de donde se aprendieron.

Con respecto a la memoria, se puede ver como está alterada en todos los niveles. La deficiencia en la memoria a corto plazo (visual y auditivo): para captar y memorizar imágenes de objetos, listados de palabras, listados de números, etc. (Bilovsky y Share, 1963; Bower y Hayes, 1994). Este tipo de memoria, en estos sujetos, no aumenta con la edad a la velocidad con que lo hace el resto de la población, sucede de forma más lenta. En cambio, en memoria visual presentan una mejoría (Marcell y Weeks, 1988). Estas dificultades suelen ser muy evi- 
dentes en el niño pequeño pero también se observan en el adolescente y en el adulto (Flórez y Ruiz, 2004).

En la memoria a largo plazo, existen claros problemas de consolidación, bien por falta de atención o de motivación. Aunque también se han podido observar carencias intrínsecas en las conexiones interneuronales y deficiencias en ciertos núcleos y áreas del cerebro (por ejemplo, el hipocampo) (Flórez, 1999). Son varias las teorías que afirman que los niños con $\mathrm{SD}$, mejoran en memoria visual, tras una fase de entrenamiento (Calero, Robles y García, 2010), pero a pesar de este punto fuerte en los niños con SD, los DA están más desarrollados en este tipo de tareas. Y en procesos de ejecución están también por debajo de los DA (Miller, Leddy y Leavitt, 2001).

El lenguaje en niños con SD, es una de las áreas más alteradas. La adquisición del lenguaje puede llegar a ser grave, pero hay casos en que la capacidad lingüística de estos niños están dentro de lo normal e incluso superior a niños sin esta dificultades. (Rondal, Perera, Nadel, y Comblain, 1997). Cuando hablamos de conducta prelingüística, los niños con SD muestran rasgos normales, adquieren el balbuceo o la imitación, pero más lentamente, (Rondal y Lang, 2009), y existen claras diferencias con respecto a niños que no presentan SD. Por ejemplo, la adquisición de palabras es más lenta, aunque vocalizan sin dificultad, también se puede apreciar, un deterioro al inicio de la producción del habla alrededor de los 2 o 3 años. En niños más mayores, la producción de primeras palabras, pragmática y comunicación, comprensión y producción, también sufre un gran retroceso (Rondal, 2001).

Las causas de las dificultades que sufren los niños con SD en el habla y el lenguaje son entre otros: los problemas del aprendizaje debido a su discapacidad intelectual, diferencias anatómicas en las que se ven afectadas las habilidades del habla, problemas auditivos (más del 50\%) debido en su mayoría a infecciones, problemas visuales como miopía y cataratas, problemas en la memoria a corto plazo, dificultades motoras en el habla, problemas en la atención, entre otros (Buckley y Bird, 2005).

A pesar de estas limitaciones, en los niños con SD las habilidades de interacción y comunicación no verbal no están tan deterioradas e incluso se ha llegado a observar, como el uso de gesto y la ayuda de estímulos visuales facilitan el desarrollo del lenguaje (Buckley y Bird, 2005). Por tanto la comunicación de tipo gestual es uno de sus puntos fuertes, (Galeote, Soto, Serrano, Pulido, Rey y Martínez-Roa, 2006) e incluso el número de producción de gestos esta por encima de los niños con desarrollo sin dificultades. (Berglund, Eriksson, Johans- 
son y Parental, 2001). Algunos autores (Rondal, Perera, Nadel, y Comblain, 1997) mantienen la hipótesis de que la evolución del lenguaje en niños con SD no es una evolución normal pero más lenta, sino que hay diferencias con niños que no sufren retraso en el lenguaje, así como con niños con otras dificultades de aprendizaje, y por tanto es una evolución exclusiva de los niños con SD, debido a los problemas genéticos de este trastorno.

\section{Objetivos e hipótesis}

El objetivo principal de este trabajo consiste en comparar qué áreas del desarrollo neuropsicológico están afectadas en nuestros grupos de niños con SD y niños con DA, con dificultades en la adquisición del lenguaje, que reciben Atención Temprana, y ver, además, en qué áreas de desarrollo difieren, a nivel general, es decir, desarrollo verbal, desarrollo no verbal, desarrollo total y coeficiente de Desarrollo y a nivel concreto en cada una de las variables del CUMANIN: psicomotricidad, lenguaje articulatorio, lenguaje expresivo, lenguaje comprensivo, estructuración espacial, visopercepción, memoria icónica, ritmo, fluidez verbal, atención, lectura y dictado. Y por tanto poder obtener diferencias significativas en el lenguaje. Igualmente se pretende descubrir si existen diferencias significativas con respecto a un grupo de niños sin diagnóstico.

Las hipótesis de trabajo planteadas en esta investigación son que existen diferencias significativas entre:

1. Las puntuaciones de todas las variables de la escala entre todos los grupos de niños, (Sin diagnóstico, SD y DA)

2. El grupo Sin diagnóstico comparado con el grupo SD y grupo con DA para cada una de las variables.

2.1. El grupo de niños con SD y el grupo de DA para cada una de las variables.

3. Cada una de las variables totales (DV, DNV, CD, y DT).

4. El grupo Sin diagnóstico comparado con el grupo SD y DA para cada una de las variables totales.

4.1. Los grupos SD y DA en las diferentes variables totales. 


\section{Método}

\section{Participantes}

La muestra está formada por un total de 51 niños y este a su vez se divide en tres grupos: uno con Síndrome de Down con edades de entre tres y seis años y de ambos sexos, otro grupo formado por niños con dificultades de aprendizaje, concretamente con problemas en la adquisición del lenguaje, de la misma edad. Ambos pertenecientes al programa de AT de la Asociación Síndrome de Down de Jaén, que previamente han sido diagnosticados por un neuropediatra. El grupo de niños diagnosticados con Síndrome de Down está formado por 11 niños, (4 niños y 7 niñas), y el grupo de niños con dificultades de aprendizaje son igualmente 11 niños (8 niños y 3 niñas).

Además de estos dos grupos de niños, hay un último grupo sin diagnosticar, ya que fue proporcionado por sus respectivos maestros, quienes consideran que son niños sin ninguna problemática aparente, con un ritmo normal dentro de su clase. Está formado por veintinueve niños, tomados de diversos colegios de la provincia de Jaén. (16 niños y 13 niñas, de entre tres y seis años de edad)

\section{Instrumento}

Para poder comprobar las hipótesis se ha utilizado el Cuestionario de Madurez Neuropsicológica Infantil, CUMANIN (Portellano, Mateos, y Martínez, 2002), siendo unos de los cuestionarios que más se han utilizado en neuropsicología y que permite identificar posibles trastornos madurativos que afectan a las funciones cognitivas del los niños, y en especial los causados por algún tipo de disfunción del sistema nervioso. Se aplica a niños de 3 a 6 años y permite detectar de forma sencilla, posibles dificultades del desarrollo en esta etapa. Las áreas que evalúa son: Psicomotricidad, Lenguaje, Atención, Estructuración espacial, Visopercepción, Memoria, Estructuración rítmico-temporal y Lateralidad. Está formado por 83 ítems y este a su vez por diferentes subescalas (13). De todas estas escalas 8 son las principales y las 5 adicionales. Las escalas principales son: psicomotricidad, lenguaje articulatorio, lenguaje expresivo, lenguaje comprensivo, estructuración espacial, visopercepción, memoria icónica y ritmo. Escalas adicionales: fluidez verbal, atención, lectura, dictado y lateralidad. 
A partir de los ítems de la escalas principales se obtiene la puntuación total, que corresponde al Coeficiente de Desarrollo Global del niño, y éste su vez al ser interpretado en centiles, se corresponde con el Cociente de Desarrollo (CD). Además con la prueba también se consigue una puntuación de desarrollo verbal (DV) y otra para el desarrollo no verbal (DNV). La duración aproximada de la prueba es de entre 30 a 50 minutos.

La fiabilidad del cuestionario es considerada por los autores como aceptable, estando fundamentada en estudios basados en análisis estadísticos con una muestra de 803 niños. En ellos se obtuvieron valores del coeficiente del alfa de Cronbach de todo el test que oscilaron entre .83 y .98 según los grupos de edad, y entre .57 y .92 en el caso de las diferentes subescalas (memoria icónica y lenguaje articulatorio respectivamente).

\section{Procedimiento}

En primer lugar se realizó una toma de contacto con los niños durante unos días. Para ello, se observó el trabajo de los profesionales de la asociación, durante las sesiones de intervención dentro del programa de Atención Temprana. En el este programa los niños reciben atención logopedica, psicológica y apoyo en las necesidades educativas. El proceso de evaluación se llevó a cabo de forma individual y con la previa autorización por parte de los padres de todos los niños.

\section{Análisis de datos}

Se ha realizado un diseño cuasiexperiemental con dos grupos equivalentes que se ha establecido dependiendo del diagnóstico: el grupo Down, y el grupo DA, junto con un grupo Sin Diagnóstico. Por tanto, la variable independiente es el diagnostico que se ha dividido en tres grupos. Síndrome de Down, dificultades de aprendizaje, y un grupo llamado Sin Diagnóstico. Las variables dependientes son las diferentes subcategorías en las que se divide el cuestionario.

Para determinar los diferentes objetivos se ha realizado un análisis descriptivo de los grupos que componen la muestra, en todas las escalas que componen el CUMANIN, se ha comprobado la homogeneidad de las varianzas para cada escala. Como las varianzas no eran homogéneas en todas las variables debido al tamaño de la muestra, se ha realizado un análisis de varianza o ANOVAS de un factor con medidas repetidas, para determinar las diferentes diferencias en cada grupo, utilizando la prueba de Greenhouse-Geisser. Posteriormente se han 
realizado comparaciones múltiples con las pruebas post hoc, utilizando los estadísticos de HSD de Tukey. Y por último se han añadido las diversas gráficas. Para llevar a cabo todos estos análisis estadísticos se ha utilizado el paquete estadístico SPSS 17.0.

\section{Resultados}

Con respecto a la edad no existen diferencias significativas entre los tres grupos de niños que componen la muestra, $F(2,48)=3.171, \mathrm{p}>.05$. Ver tabla 1 .

Tabla 1 distribución de la variable edad de cada grupo.

\begin{tabular}{lcccc}
\hline Grupos & $\begin{array}{c}\text { Rango } \\
\text { de edad }\end{array}$ & $\mathrm{N}$ & media & $\mathrm{dt}$. \\
\hline $\begin{array}{l}\text { Sin dia- } \\
\text { gnóstico }\end{array}$ & $3-6$ & 29 & 53.72 & $(11.44)$ \\
\hline Down & $3-6$ & 11 & 57.90 & $(13.17)$ \\
\hline DA & $3-6$ & 11 & 46.09 & $(8.34)$ \\
\hline
\end{tabular}

En lo que se refiere a la hipótesis 1, si existen diferencias significativas en cada una de las variables, los resultados obtenidos en todas las subcategorías que componen el cuestionario, $($ Lambda de Wilks $=.141 ; \mathrm{F}=(11,38)=20.97 ; \mathrm{p}=.00)$ para todas las variables y (Lambda de Wilks $=.190 ; \mathrm{F}=(22,76)=4.48 ; \mathrm{p}=.00)$ para la interacción entre las variables y diagnostico. En la tabla 2 se pueden observar las puntuaciones que se han obtenido en las distintas variables que forman el CUMANIN, para cada grupo. Por tanto se puede afirmar que se cumple la hipótesis. 
Tabla 2. Puntuaciones obtenidas por los grupos para cada variable.

\begin{tabular}{|c|c|c|c|c|c|}
\hline Grupos & Variable & Mínimo & Máximo & Media & Dt. \\
\hline \multirow{12}{*}{$\begin{array}{l}\text { Sin dia- } \\
\text { gnóstico }\end{array}$} & Psicomotricidad & 5.00 & 11.00 & 8.06 & $(1.53)$ \\
\hline & $\begin{array}{l}\text { Lenguaje arti- } \\
\text { culatorio }\end{array}$ & .00 & 15.00 & 10.03 & $(3.85)$ \\
\hline & $\begin{array}{l}\text { Lenguaje ex- } \\
\text { presivo }\end{array}$ & .00 & 4.00 & 2.48 & $(1.35)$ \\
\hline & $\begin{array}{l}\text { Lenguaje com- } \\
\text { prensivo }\end{array}$ & .00 & 9.00 & 4.44 & $(2.77)$ \\
\hline & $\begin{array}{l}\text { Estructuración } \\
\text { espacial }\end{array}$ & 4.00 & 14.00 & 8.41 & $(2.95)$ \\
\hline & Visopercepción & 1.00 & 14.00 & 7.86 & (3.93) \\
\hline & $\begin{array}{l}\text { Memoria iconi- } \\
\text { ca }\end{array}$ & 2.00 & 9.00 & 5.79 & (1.54) \\
\hline & Ritmo & .00 & 7.00 & 2.72 & (1.70) \\
\hline & Fluidez verbal & .00 & 42.00 & 11.34 & $(12.29)$ \\
\hline & Atención & .00 & 20.00 & 9.75 & $(5.73)$ \\
\hline & Lectura & .00 & 11.00 & 2.20 & $(4.10)$ \\
\hline & Dictado & .00 & 61.00 & 3.72 & (11.47) \\
\hline \multirow{15}{*}{ Down } & Psicomotricidad & 1.00 & 9.00 & 3.54 & $(2.29)$ \\
\hline & $\begin{array}{l}\text { Lenguaje arti- } \\
\text { culatorio }\end{array}$ & .00 & 3.00 & .63 & (1.02) \\
\hline & $\begin{array}{l}\text { Lenguaje ex- } \\
\text { presivo }\end{array}$ & .00 & .00 & .00 & $(.00)$ \\
\hline & $\begin{array}{l}\text { Lenguaje com- } \\
\text { prensivo }\end{array}$ & .00 & 3.00 & 1.27 & (1.27) \\
\hline & $\begin{array}{l}\text { Estructuración } \\
\text { espacial }\end{array}$ & .00 & 10.00 & 4.09 & $(3.17)$ \\
\hline & Visopercepción & .00 & 9.00 & 1.54 & $(2.73)$ \\
\hline & $\begin{array}{l}\text { Memoria iconi- } \\
\text { ca }\end{array}$ & .00 & 7.00 & 2.09 & $(2.58)$ \\
\hline & Ritmo & .00 & 1.00 & .36 & $(.50)$ \\
\hline & Fluidez verbal & .00 & .00 & .00 & $(.00)$ \\
\hline & Atención & .00 & 15.00 & 5.00 & $(5.98)$ \\
\hline & Lectura & .00 & 10.00 & 1.54 & $(3.50)$ \\
\hline & Dictado & .00 & 1.00 & .09 & $(.30)$ \\
\hline & Psicomotricidad & 1.00 & 7.00 & 3.36 & $(1.80)$ \\
\hline & $\begin{array}{l}\text { Lenguaje arti- } \\
\text { culatorio }\end{array}$ & & 8.00 & .90 & $(2.38)$ \\
\hline & $\begin{array}{l}\text { Lenguaje ex- } \\
\text { presivo }\end{array}$ & & 1.00 & .09 & $(.30)$ \\
\hline
\end{tabular}




\begin{tabular}{|c|c|c|c|c|c|}
\hline & $\begin{array}{l}\text { Lenguaje com- } \\
\text { prensivo }\end{array}$ & .00 & 6.00 & 1.81 & $(2.27)$ \\
\hline \multirow[t]{8}{*}{ DA } & $\begin{array}{l}\text { Estructuración } \\
\text { espacial }\end{array}$ & .00 & 9.00 & 4.00 & $(2.60)$ \\
\hline & Visopercepción & .00 & 3.00 & .90 & (1.37) \\
\hline & $\begin{array}{l}\text { Memoria icóni- } \\
\text { ca }\end{array}$ & 1.00 & 7.00 & 4.09 & $(2.11)$ \\
\hline & Ritmo & .00 & 4.00 & .81 & $(1.25)$ \\
\hline & Fluidez verbal & .00 & 2.00 & .18 & $(.60)$ \\
\hline & Atención & .00 & 20.00 & 5.36 & $(6.84)$ \\
\hline & Lectura & .00 & .00 & .00 & $(.00)$ \\
\hline & Dictado & .00 & .00 & .00 & $(.00)$ \\
\hline
\end{tabular}

En la hipótesis 2, hubo que comprobar si existían diferencias significativas entre los niños Sin Diagnóstico y SD, e igualmente, si existen diferencias significativas entre el grupo Sin diagnostico y DA, en cada una de las variables. Según los resultados post hoc realizados y con una significación de $<0.05$, existen diferencias significativas a favor del grupo Sin Diagnóstico en la mayoría de las subescalas del cuestionario (psicomotricidad, lenguaje articulatorio, lenguaje expresivo, lenguaje comprensivo, estructuración espacial, visopercepción, ritmo y fluidez verbal. Los resultados obtenidos fueron los siguientes: Psicomotricidad: $(\mathrm{DM}=4.52 ;$ E.T. $=.62 ; \mathrm{p}=.00)$ para Sin diagnostico y SD y $(\mathrm{DM}=4.70 ; \mathrm{E} . \mathrm{T} .=.62 ; \mathrm{p}=.00)$ para Sin diagnostico y DA. Lenguaje articulatorio $(\mathrm{DM}=9.39$; E.T. $=1.12 \mathrm{p}=.00)$ para Sin diagnostico y SD y $(\mathrm{DM}=9.12$; E.T.=.1.12; p=.00) para Sin diagnostico y DA. Lenguaje expresivo: $(\mathrm{DM}=2.48 ;$ E.T.=.36; p=.00) para Sin diagnostico y SD y $(\mathrm{DM}=2.39 ;$ E.T.=.36; $\mathrm{p}=.00)$ para Sin diagnostico y DA. Lenguaje compresivo: $(\mathrm{DM}=3.17$; E.T. $=.85 ; \mathrm{p}=.002)$ para Sin diagnostico y SD y $(\mathrm{DM}=2.63 ;$ E.T.=.85; p=.01) para Sin diagnostico y DA. Estructuración Espacial: $(\mathrm{DM}=4.32$; E.T.=1.03; p=.00) para Sin diagnostico y SD y $(\mathrm{DM}=4.41$; E.T.=.1.03; $\mathrm{p}=.00)$ para Sin diagnostico y DA. Visopercepción: $(\mathrm{DM}=6.31$; E.T. $=.1 .17$; $=.00)$ para Sin diagnostico y SD y (DM=6.95; E.T.=.1.17; p=.00) para Sin diagnostico y DA. Memoria Icónica: $(\mathrm{DM}=3.70 ;$ E.T.=.68; $\mathrm{p}=.00)$ para Sin diagnostico y SD y $(\mathrm{DM}=1.70 ;$ E.T.=.68; $\mathrm{p}=.042)$ para Sin diagnostico y DA. Ritmo: $(\mathrm{DM}=2.36$; E.T.=.51; $\mathrm{p}=.00)$ para Sin diagnostico y SD y $(\mathrm{DM}=1.90 ;$ E.T. $=.51 ; \mathrm{p}=.001)$ para Sin diagnostico y DA. Fluidez verbal: $(\mathrm{DM}=11.34$; E.T. $=3.32 ; \mathrm{p}=.004)$ para Sin diagnostico y SD y $(\mathrm{DM}=11.16$; E.T.=3.32; $=.004)$ para Sin diagnostico y DA. Para el resto de las variables (Atención, lectura y dictado) no existen diferencias significativas entre Sin diagnóstico y SD y Sin diagnóstico y DA. 


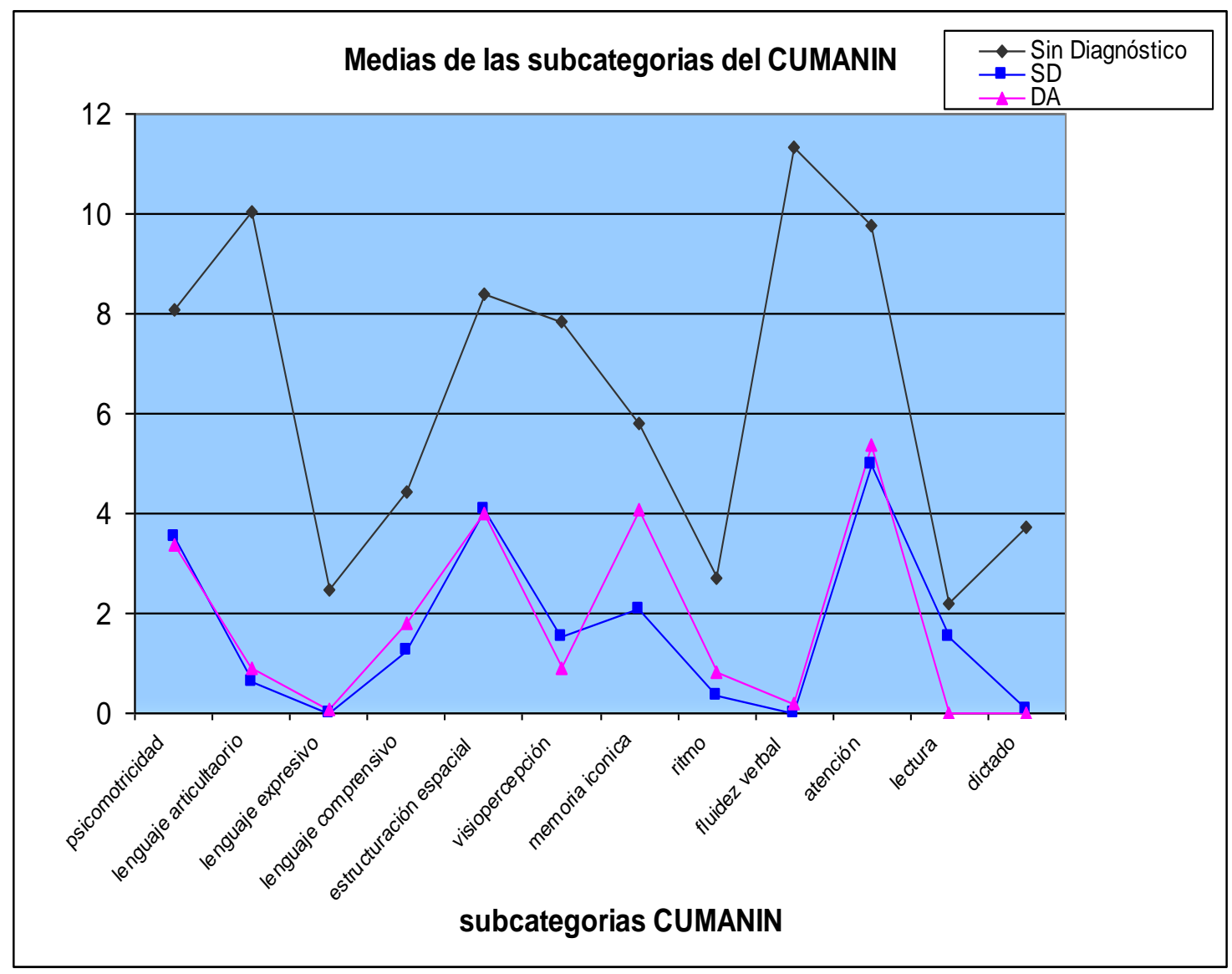

Figura 1. Grafica de las subcategorías que forman el CUMANIN

Con respecto a la hipótesis 2.1, existen diferencias significativas entre el grupo SD y DA para cada una de las variables. Los resultados obtenidos fueron los siguientes: No hay diferencias significativas en las variables psicomotricidad, lenguaje articulatorio, lenguaje expresivo, lenguaje comprensivo, estructuración espacial, visopercepción, ritmo, fluidez verbal, atención lectura, y dictado, entre el grupo SD y DA. En la única variable en la que si existen diferencias significativas es en memoria icónica $(\mathrm{DM}=-2.00 ; \mathrm{ET}=.822 ; \mathrm{p}=.048)$. 


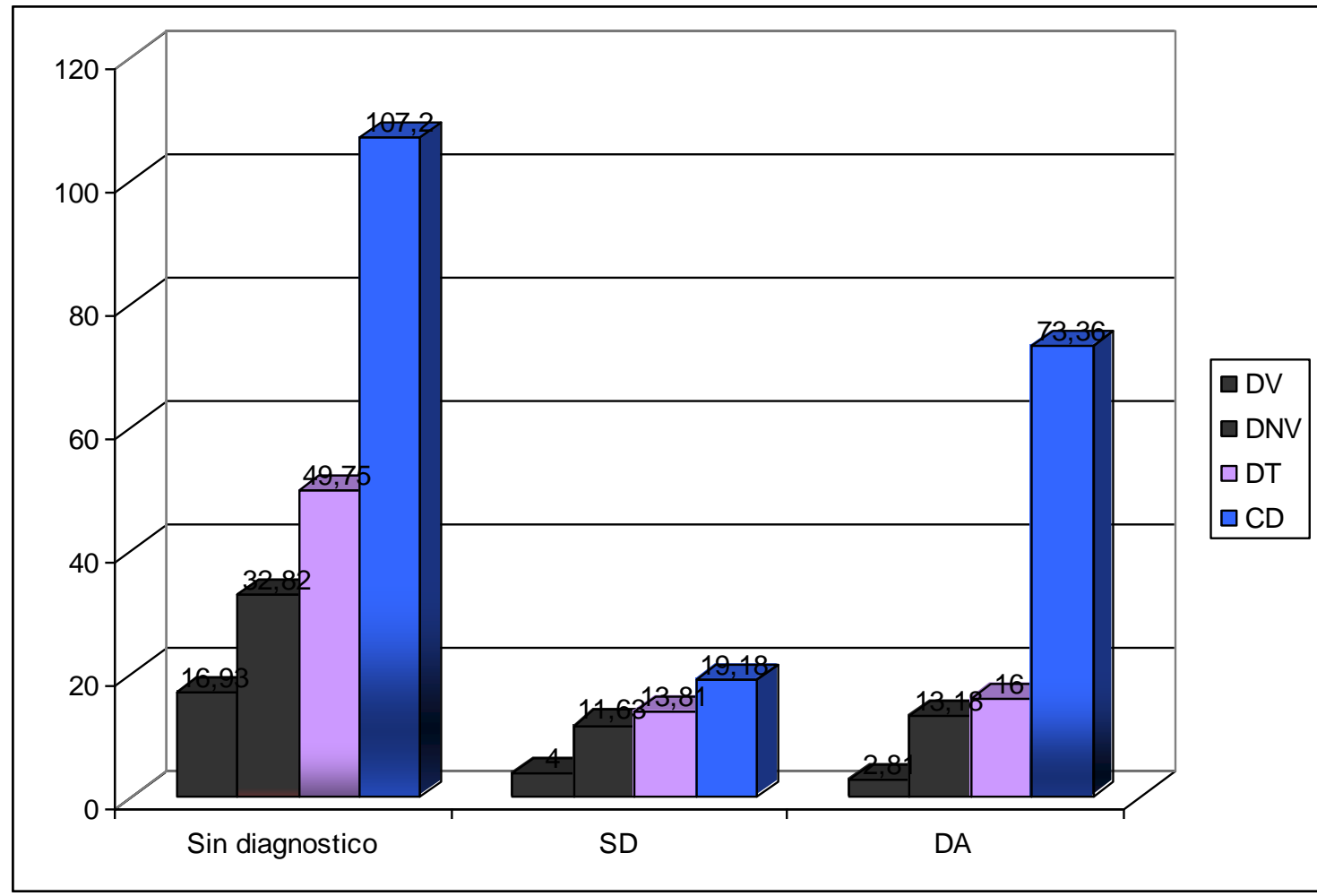

Figura 2. Medias para cada variable totales (desarrollo verbal (DV) desarrollo no verbal (DNV), desarrollo total y coeficiente de desarrollo)

Si se comprueba la hipótesis 3, existen diferencias significativas entre cada una de las variables totales del CUMANIN, los resultados obtenidos en las variables totales, aparecen diferencias significativas (Lambda de Wilks $=.083$; $F=(3,46)=169.151 ; \mathrm{p}=.00)$ y $($ Lambda de Wilks $=.126 ; \mathrm{F}=(6,92)=27.78 ; \mathrm{p}=.00)$ para la interacción entre las variables y diagnostico. Por tanto, existen diferencias significativas entre los tres grupos de niños que forman la muestra (Tabla 3).

Tabla 3. Puntuaciones obtenidas por los grupos para las variables totales (desarrollo verbal (DV) desarrollo no verbal (DNV), desarrollo total y coeficiente de desarrollo)

\begin{tabular}{lllllll}
\hline Grupos & Variable & Mínimo & Máximo & Media & Dt. \\
\hline & $\begin{array}{l}\text { Desarrollo ver- } \\
\text { bal }\end{array}$ & 1.00 & 25.00 & 16.93 & $(6.79)$ \\
\cline { 2 - 6 } $\begin{array}{l}\text { Sin dia- } \\
\text { gnóstico }\end{array}$ & $\begin{array}{l}\text { Desarrollo } \\
\text { verbal }\end{array}$ & no & 20.00 & 49.00 & 32.82 & $(8.81)$ \\
\cline { 2 - 6 } & Desarrollo total & 24.00 & 74.00 & 49.75 & $(14.13)$
\end{tabular}




\begin{tabular}{|c|c|c|c|c|}
\hline & $\begin{array}{lll}\text { Coeficiente de } & 79.00 \\
\text { desarrollo } & & \\
\end{array}$ & 130.00 & 107.20 & $(11.86)$ \\
\hline \multirow{4}{*}{ SD } & $\begin{array}{lll}\text { Desarrollo } & \text { ver- } & .00 \\
\text { bal } & & \end{array}$ & 22.00 & 4.00 & $(6.26)$ \\
\hline & $\begin{array}{lll}\text { Desarrollo no } & 2.00 \\
\text { verbal } & & \end{array}$ & 35.00 & 11.63 & $(10.41)$ \\
\hline & Desarrollo total 2.00 & 40.00 & 13.81 & $(11.88)$ \\
\hline & $\begin{array}{lll}\text { Coeficiente de } & .00 \\
\text { desarrollo } & & \\
\end{array}$ & 79.00 & 19.18 & $(33.06)$ \\
\hline \multirow{4}{*}{ DA } & $\begin{array}{lll}\text { Desarrollo } & \text { ver- } & .00 \\
\text { bal } & & \end{array}$ & 15.00 & 2.81 & $(4.49)$ \\
\hline & $\begin{array}{lll}\begin{array}{l}\text { Desarrollo no } \\
\text { verbal }\end{array} & & \\
\text { verbon }\end{array}$ & 25.00 & 13.18 & (7.50) \\
\hline & Desarrollo total 5.00 & 40.00 & 16.00 & $(11.16)$ \\
\hline & $\begin{array}{l}\text { Coeficiente de } 65.00 \\
\text { desarrollo }\end{array}$ & 88.00 & 73.36 & $(7.65)$ \\
\hline
\end{tabular}

En la hipótesis 4, hay que comprobar si existen diferencias significativas entre los niños Sin Diagnóstico y SD, e igualmente, si existen diferencias significativas entre el grupo Sin diagnostico y DA, en cada una de las variables totales (Desarrollo verbal, Desarrollo no verbal, Coeficiente de Desarrollo y Desarrollo Total). Desarrollo Verbal cuyo resultado obtenido fue de $(\mathrm{DM}=12.93 ; \mathrm{ET}=2.22$ p=.00) para Sin diagnóstico y Down y de $(\mathrm{DM}=14.11$; $\mathrm{ET}=2.22 ; \mathrm{p}=.00)$ para Sin diagnóstico DA. Desarrollo no verbal los resultados obtenidos fueron $(\mathrm{DM}=21.19 ; \mathrm{ET}=3.16 ; \mathrm{p}=.00)$ para Sin diagnóstico y Down y de $(\mathrm{DM}=19.64 ; \mathrm{ET}=3.16$; $\mathrm{p}=.00$ ) para Sin diagnóstico y DA. Desarrollo Total los resultados obtenidos fueron $(\mathrm{DM}=35.94 ; \mathrm{ET}=4.64 ; \mathrm{p}=.00)$ para sin diagnóstico y Down y de $(\mathrm{DM}=33.75 ; \mathrm{ET}=4.64$; p=.00) para Sin Diagnóstico y DA. Para el Coeficiente de Desarrollo los resultados obtenidos fueron $(\mathrm{DM}=88.02$; $\mathrm{ET}=6.35 ; \mathrm{p}=.00)$ para Sin Diagnóstico y Down y de $(\mathrm{DM}=33.84$; $\mathrm{ET}=6.35 ; \mathrm{p}=.00)$ para Sin diagnóstico y DA. Por tanto, se puede concluir que existen diferencias significativas en las 4 variables totales a favor de los niños Sin diagnóstico.

En cuanto a la Hipótesis 4.1. (Existen diferencias entre las variables totales en niños con SD y niños con DA), tanto para el desarrollo verbal como para el no verbal, no existen 
diferencias significativas entre ambos grupos. En Desarrollo Total los resultados obtenidos según las pruebas post-hoc no existen diferencias entre ambos grupos. Para el Coeficiente de Desarrollo los resultados obtenidos fueron $(\mathrm{DM}=-54.18 ; \mathrm{ET}=7.64 ; \mathrm{p}=.00)$ para ambos grupos. Por tanto, hay diferencias significativas en el Coeficiente de Desarrollo entre niños con SD y niños con DA. (Figura 2)

\section{Discusión y conclusiones}

El objetivo del presente estudio, es comparar a través del cuestionario CUMANIN, las áreas del desarrollo neuropsicológico afectadas en niños con Síndrome de Down y con Dificultades del Aprendizaje para poder comprobar en qué áreas difieren, igualmente comparamos estos grupos con un grupo de niños Sin diagnóstico y poder así tener una referencia sobre el desarrollo evolutivo normal.

Con respecto al primer objetivo, podemos afirmar que no existen diferencias significativas debidas a la edad.

En cuanto a las siguientes hipótesis, podemos afirmar que existen diferencias significativas en la totalidad de las variables dependientes (subcategorías) a favor de los niños Sin diagnostico. Las puntuaciones medias obtenidas por el grupo Sin diagnostico, tal y como se esperaba, son superiores a las medias obtenidas por los otros dos grupos de sujetos en las que las diferencias encontradas, no son significativas, al contrario de lo que aparece en la literatura científica, como ocurre en el área del lenguaje (Laws y Bishop, 2003), en memoria declarativa (Nadel, 2000), problemas a nivel atencional, procesamiento de la información, en memoria a corto plazo, más dificultades para resolver problemas (Chapman y Hesketh, 2000), (Flórez, 1999), etc. Por tanto hay mayor interacción en sus puntuaciones medias y esto nos indica que la ejecución de los sujetos con SD y sujetos con DA es similar, a pesar de que como se ha comentado se esperaba lo contrario, es decir, los niños con SD tradicionalmente aparecen por debajo de los niños con DA en ejecución. (Sastre, 2006). Las resultados no son significativos tanto en psicomotricidad, como en lenguaje articulado, expresivo, como comprensivo, estructuración espacial, visopercepción, ritmo, atención, lectura, dictado y fluidez verbal. En las subcategorías de lectura y en escritura no existen diferencias entre los tres grupos y por tanto puntúan de forma similar ya que están en proceso de aprendizaje. 
En cambio, en la única variable en la que existen diferencias significativas entre niños SD y DA, es la Memoria Icónica, resultados que demuestran que los niños con DA responden mejor que los niños con SD, aunque los niños con SD también obtienen mejores resultados en esta variable, que en el resto de las subcategorías, sin embargo aparece por debajo. Este resultado coinciden con las de estudios previos que demuestran, que este tipo de memoria, es una de las ventajas que poseen estos niños (Calero, Robles y García, 2010), y por ello, se podría aprovechar esta circunstancia a favor tanto en niños con DA, como con SD para una mejora del desarrollo en el lenguaje. En este contexto, se podría proponer tratamientos basados en sistemas de comunicación alternativos y utilizando para su aprendizaje, la vía visual o memoria visual por medio de imágenes, pictogramas o signos visuales. Son varios los estudios que indican que la enseñanza de signos o símbolos facilitan la adquisición del lenguaje oral en niños con dificultades graves en lenguaje, y no lo retrasa, como se puede llegar a pensar (Carr y Dores, 1981), o también utilizando estos mismos métodos visuales, para la enseñanza de la lectura (Comes, 2006; Comes, Aznar, Contijoch, y Vives, 2001; Daza et al., 2011; Troncoso y del Cerro, 1998), en niños desde edades muy tempranas.

En cuanto a los resultados de las puntuaciones totales, la hipótesis 4 y 5, no se han encontrado diferencias significativas en el área del lenguaje (ya sea verbal como no verbal) y por tanto los niños con Síndrome de Down como los DA están igual de afectados en ambas variables, pero si hay diferencias significativas con respecto al grupo Sin Diagnóstico. En Desarrollo Total, la suma de todas las puntuaciones que forman el CUMANIN, los resultados obtenidos muestran que no existen diferencias entre ambos grupos SD y DA. Pero si hay diferencias de ambos respecto al grupo Sin Diagnóstico, a favor de este último.

En la variable en la que si se han encontramos diferencias significativas entre los tres grupos de sujetos es el Coeficiente de Desarrollo (recordemos que es un índice obtenido a partir del índice de Desarrollo Total y es comparable al Cociente de Inteligencia). Por tanto, todos los grupos puntúan de forma diferente, estando mejor por este orden Sin diagnóstico, DA y SD. Estos resultados apoyan las hipótesis de las investigaciones, en las que se pone de de manifiesto que los niños con SD muestra un coeficiente intelectual muy por debajo de otros niños con otras dificultades del aprendizaje (Rondal, Perera, Nadel y Comblain, 1997)

¿Podemos afirmar que el Cuestionario de Madurez Neuropsicológica Infantil, CUMANIN, nos ayuda a determinar qué áreas están afectadas entre ellas el lenguaje, en niños con diversas dificultades de aprendizaje, como en el caso de los niños de este estudio? Real- 
mente no queda tan claro. Si es cierto que ayuda a diferenciarlos de un grupo de sujetos Sin Diagnosticar, pero a la hora de evaluar a los niños con DA y con SD no se encuentran diferencias a nivel cognitivo de un grupo y otro, y por tanto se puede cuestionar su eficacia. También se puede pensar que estos niños al estar juntos en un programa de Atención Temprana juntos, en la misma asociación, estén recibiendo un entrenamiento en habilidades cognitivas similares y por tanto se estén obteniendo resultados parecidos en ambos grupos.

Habría que seguir investigando y poder ampliar la muestra para llegar a obtener resultados más concluyentes, ya que la muestra, como ya se ha dicho anteriormente, es muy pequeña debido al rango de edad, y al tipo de dificultad, o bien se podrían realizar seguimientos posteriores de estos mismos niños, y realizar las comparaciones pertinentes para obtener mejores resultados. 


\section{Referencias}

Arregi, A. (1996). Síndrome de Down: necesidades educativas y desarrollo del lenguaje. Dirección de Renovación Pedagógica. Instituto para el desarrollo Curricular y la formación del profesorado. Área de Necesidades Educativas Especiales. Vitoria-Gasteiz: Servicio Central de Publicaciones del Gobierno Vasco.

Asociación Psiquiátrica Americana (1995). Manual diagnóstico y estadístico de los trastornos mentales DSM-IV-TR. Ed. Masson. Barcelona.

Berglund E., Eriksson M., Johansson, I. Parental (2001). Reports of spoken language skills in children with Down syndrome. Journal of Speech, Language, and Hearing Research, $44,179-191$.

Bilovsky, D., Share, J. (1965). The ITPA and Down syndrome: an exploratory study. American Journal of Mental Deficiency, 70, 78-82.

Bisquerra, R. (2000). Educación emocional y bienestar. Ed. Praxis. Barcelona

Bower, A., Hayes, A. (1994). Short term memory deficits and Down syndrome: a comparative study. Down's syndrome: Research and Practice, 2, 47-50.

Buckley S., Bird G., Perera J. (2005). Habla, lenguaje y comunicación en alumnos con síndrome de Down. Ed. CEPE, Madrid.

Calero, M.D., Robles, M. A. García, B. (2010). Habilidades cognitivas, conducta y potencial de aprendizaje en preescolares con síndrome de Down. Electronic Journal of Research in Educational Psychology, 8(1), 87-110.

Carr, E. G., Dores, P. A. (1981). Patterns of language acquisition following simultaneous communication with autistic children. Analysis and Intervention in Developmental Disabilities, 1, 347-361.

Chapman, R.S., Hesketh, L. J. (2000). Behavioral phenotype of individuals with Down Syndrome. Mental Retardation and Developmental Disability. Research Reviews, 6, 8495.

Comes, G., Aznar, C., Contijoch, T. y Vives, M. (2001). Enseñanza inicial de la lectura en niños y niñas con Síndrome de Down. Bordón, 53(1), 21-28.

Comes, G. (2006). Enseñar a leer al alumnado con Síndrome de Down. Programas de intervención temprana. Ed. Aljibe.

Daza, M. T., Guil, F. G., López, F., Salmerón, R., García, N. (2011, Septiembre). Evaluación Neuropsicológica en niños sordos: Resultados preliminares obtenidos con la batería 
AWARD Neuropsychological. Electronic Journal of Research in Educational Psychology, 9(2), 849-868.

Flórez, J. y Ruiz, E. (2004). El síndrome de Down: aspectos biomédicos, psicológicos educativos. Disponible (online): htto://www.down21.com/vision_perspec /aspectos _biomedicos.htm.

Flórez J. (1999). Patología cerebral y sus repercusiones cognitivas en el síndrome de Down. Siglo Cero. 30(3), 29-45.

Flórez, J. (1999). Bases neurobiológicas del aprendizaje. Siglo Cero. 30(183), 9-27

Galeote, M.; Soto, P.; Serrano, A.; Pulido, L.; Rey, R. y Martínez-Roa, P. (2006). Un nuevo instrumento para evaluar el desarrollo comunicativo y lingüístico de niños con síndrome de Down. Revista Síndrome de Down, 88, 20-26.

Hasson, N. y Joffe, V. (2007). The case for Dynamic Assessment in speech and language therapy. Child Language Teaching and Therapy, 23(1), 9-25.

Laws. G. y Bishop, D.V.M. (2003). A comparison of language abilities in adolescents with Down Syndrome and children with specific language impairment. Journal of Speech, Language and Hearing Research, 46, 1324-1339.

Marcell, M. M. y Weeks, S. L. (1988). Short-term memory difficulties and Down's syndrome. Journal of Mental Deficiency Research, 32, 153-162.

Miller J. F, Leddy M. y Leavitt L. A. (2001). Síndrome de Down: comunicación, lenguaje, habla. Ed. Masson y Fund. Síndrome de Down de Cantabria. Barcelona

Miranda, A., Vidal-Abarca, E., Soriano, M. (2000). Evaluación e intervención psicoeducativa en dificultades de aprendizaje. Ed. Pirámide. Madrid.

Nadel, L. (2000). Aprendizaje y memoria en el Síndrome de Down. En Rondal, L.; Perera, L.; Nadel, L. (Coords). Síndrome de Down, revisión de los últimos conocimientos( pp. 197-209). Madrid: Espasa Calpe.

Organización Diagnostica para la Atención Temprana, (ODAT) (2008). Glosario Actualización de la Organización Diagnostica para la Atención Temprana. Federación Estatal de Asociaciones de profesionales de Atención Temprana GAT. Ministerio de Sanidad y Política Social.

Padget, S. Y. (1998). Lessons from research on dyslexia: Implications for a classification system for learning disabilities. Learning Disability Quarterly, 21, 167-176.

Portellano, J.A. y Mateos, R.; Martínez, R. (1999). CUMANIN. Cuestionario de Madurez Neuropsicológica Infantil. Madrid: TEA Ediciones, S.A. 
Rondal, J.A y Lang, SC. (2009). Atención Temprana: Comunicación y el desarrollo del lenguaje. Revista Síndrome de Down. Revista Española de Investigación e Información sobre el Sindrome de Down, 26.

Rondal, J.A (2001). El lenguaje en el retraso mental: diferencias individuales y sindrómicas y variación neurogenética. Revista de logopedia, foniatría y audiología, 21(2), 46-63

Rondal, J.A. (1984). El desarrollo del lenguaje. Ed. Médica y Técnica, S.A. Barcelona.

Rondal, J.A., Perera, J., Nadel, L. y Comblain, A. (1997). Síndrome de Down: perspectivas psicológicas, psicobiológica y socioeducacional. IMSERSO, Madrid.

Sastre-Riba, S. (2006). Condiciones tempranas del desarrollo y aprendizaje: el papel de las funciones ejecutivas. Revista de Neurología, 42(Supl. 2), 143-151

Swanson, H. L. y Howard, C. B. (2005). Children with Reading Disabilities: Does Dynamic Assessment Help in the Classification? Learning Disability Quarterly, 28, 17-36.

Troncoso M.V. y del Cerro M. (1998). Síndrome de Down: lectura y escritura. Ed. Masson.

VV.AA. (2000). Libro blanco de la atención temprana. Madrid: Real Patronato sobre Discapacidad. 